\title{
L'INTRODUCTION D'ESPÈCES ALLOCHTONES DE GRENOUILLES VERTES EN FRANCE, DEUX PROBLĖMES DIFFÉRENTS : CELUI DE R. CATESBEIANA ET CELUI DES TAXONS NON PRÉSENTS DU COMPLEXE ESCULENTA.
}

\author{
A. NEVEU \\ Laboratoire d'Ecologie Aquatique, INRA, \\ 65 rue de Saint-Brieuc, 35042 Rennes Cedex, France.
}

\section{RÉSUMÉ}

Depuis quelques années, la grenouille taureau (Rana catesbeiana) est signalée en France dans une zone limitée de gravières près de Bordeaux. Elle est aussi présente depuis peu aux Pays-Bas et, dans les deux cas, la reproduction est effective.

Cette espèce existe déjà en Europe depuis les années 30 , à la suite d'introductions volontaires en Italie, sans pour cela avoir été signalée dans la partie ouest.

La grenouille taureau est la plus grande des espèces nord-américaines et est considérée comme un prédateur éclectique. De ce fait, elle peut présenter un danger pour d'autres animaux, en particulier les grenouilles autochtones. Mais aucune donnée ne permet d'estimer cet éventuel impact en France et les données de la littérature ont souvent surestimé ce dernier. Par précaution, des mesures pourraient être prises pour réduire les populations actuelles présentes dans une aire pour le moment limitée.

Les importations de grenouilles vivantes pour la consommation humaine, surtout à partir des pays du sud de l'Europe, sont l'occasion d'introductions de nouveaux taxons. Mais ces grenouilles font partie du même complexe et sont proches sur le plan morphologique, biologique et écologique. Les risques potentiels pour les populations locales sont les introductions de gènes, mais en ce qui concerne un éventuel avantage compétitif aucune donnée ne permet de conclure.

\section{THE INTRODUCTION OF ALLOCHTONOUS GREEN FROG SPECIES INTO FRANCE, TWO DISSIMILAR CASES : $R$. CATESBEIANA AND THE FOREIGN TAXA OF THE ESCULENTA COMPLEX.}

\section{SUMMARY}

The bullfrog (Rana catesbeiana) has occurred in France for some years in a limited area of gravel pits near Bordeaux. During the same period, introduced bullfrogs were also detected in a Netherlands pond. Reproduction was recorded in both populations. The species has been living in Italy since the end of the thirties owing to voluntary introduction, though it has never been mentioned in West European records.

The bullfrog is the largest North American frog and an opportunistic predator, so it can be a threat to some other animais (native frogs, waterfowl,...). There is no data on actual impacts on the local fauna in France, and related observations were often overestimated in the literature. Precautionary measures ought to be taken to reduce numbers in its presently restricted populations. 
Live frogs imported from Southern Europe for human consumption represent another flow of foreign taxa. These frogs belong to the same green frog complex as our native specimens and are very close to them according to morphological, biological and ecological criteria. The potential risk for local populations is gene introgression, but no data suggests any competitive superiority of these foreign specimens.

\section{INTRODUCTION}

Dans le cadre des introductions d'espèces étrangères de batraciens, le cas des grenouilles vertes est le plus marquant avec deux problèmes très différents :

- celui de Rana catesbeiana (la grenouille taureau), espèce nord-américaine de grande taille,

- celui des autres taxons, non présents en France, du complexe esculenta (la grenouille comestible) constitués d'animaux relativement proches.

Mais il faudrait aussi considérer les possibles déplacements interrégionaux de certains composants de ce complexe, tout en restant à l'intérieur des frontières administratives.

A priori, le niveau des risques est très différent entre, d'une part, un élément totalement étranger, de grande taille, prédateur éclectique reconnu et, d'autre part, des grenouilles appartenant au même complexe, morphologiquement et écologiquement proches, de la même zone géographique.

\section{LE CAS DE RANA CATESBEIANA}

Cet animal a suscité l'intérêt des gastronomes depuis longtemps mais peut aussi être considéré comme prédateur d'animaux indésirables, aussi a-t-il été introduit dans plusieurs pays : Japon, Brésil, Taiwan, Cuba, Hawaï, etc.

En France, des essais d'introduction au siècle dernier ont échoué, mais par contre l'espèce s'est installée en Italie dans les années 30 pour ensuite coloniser une bonne partie de la péninsule (ALBERTINI et LANZA, 1987).

Signalée plus récemment en Hollande (1989-1990) à partir de fuites dans le circuit des amateurs de vivariums, la reproduction semble effective (STUMPEL, 1992).

En France, à la suite d'une introduction probablement volontaire, sa présence est signalée à la fin des années 80 par différents observateurs. En 1991, la présence de nombreux descendants (têtards, grenouillettes) est observée par TOURATIER (1992) dans une gravière près de Bordeaux, indiquant une acclimatation de l'espèce. Actuellement, la zone colonisée concerne surtout les gravières du nord de Bordeaux entre Garonne et Dordogne. Elle se serait récemment étendue au Médoc.

\section{Rappel de biologie et d'écologie}

R. catesbeiana est originaire de la côte est des USA depuis la frontière mexicaine jusqu'à la région des grands lacs et le sud du Canada. A la suite de diverses introductions, elle colonise actuellement la côte ouest du nord au sud et une partie du Mexique. Elle colonise ainsi une vaste zone aux climats variés, du continental au semi-tropical.

Les caractéristiques morphologiques de l'adulte se résument à :

- une robe vert jaune à brun, souvent contrastée et vermiculée,

- des tympans très développés chez le mâle,

- une taille importante pouvant atteindre $18 \mathrm{~cm}$ chez le mâle et $20 \mathrm{~cm}$ chez la femelle, pesant plusieurs centaines de grammes. 
Normalement, c'est une espèce d'étangs riches en végétation et qui reste près de l'eau. Mais elle peut coloniser tous les types de plans d'eau. C'est un animal plutôt solitaire, les mâles peuvent défendre un territoire de 3 à $25 \mathrm{~m}$ par individu (EMLEN, 1968). Si les conditions deviennent mauvaises, des migrations sont possibles.

L'activité des adultes débute lorsque la température de l'eau dépasse $13-14^{\circ}$ et celle de l'air 20-24. La ponte a lieu en mai - juin, le nombre d'oeufs variant de 1000 à 20000 par femelle, avec des records au-delà de 45000 , en fonction de la taille des géniteurs. Suivant le climat et la taille des femelies, il peut y avoir 2 pontes par an, la 2 ème étant plus faible et avec des oeufs plus petits (HOWARD, 1978). Le développement des têtards dépend de la température, de 5 mois en Louisiane, en Californie, jusqu'à 2-3 ans au Québec. La taille des têtards est normalement de 15 à $17 \mathrm{~cm}$, leur alimentation est constituée de détritus, de périphyton récolté sur les végétaux.

Après la métamorphose, la petite grenouille s'alimente surtout sur des invertébrés terrestres et aquatiques, mais dès que la taille le permet elle s'alimente sur des proies plus importantes : écrevisses, grenouilles,... La maturité sexuelle est atteinte à $9-10 \mathrm{~cm}$. A l'état adulte, elle est capable d'ingérer des petits reptiles, des rongeurs, des oiseaux,... On a même retrouvé de petits alligators dans les contenus stomacaux (BURY et WHELAN, 1984).

Les facteurs de mortalité sont variables, mais de nombreux prédateurs (invertébrés, poissons) consomment les oeufs et les têtards. A l'état adulte, elle est capturée par les rapaces, les carnassiers, les serpents,...

Si les adultes peuvent héberger un grand nombre de parasites, ils peuvent être sensibles à certaines bactéries. Ainsi, la maladie des pattes rouges (septicémie à Aeromonas, Citrobacter,...) peut détruire une partie des stocks, surtout au moment de l'hibernation ou ces bactéries psychrophiles présentes dans le tube digestif deviennent pathogènes suite à l'arrêt du transit et à la réduction des défenses immunitaires (CARR et al., 1976).

D'autres risques existent au niveau des têtards en particulier avec le développement des polluants, ceux-ci étant entre autres particulièrement sensibles aux pesticides.

\section{Interactions avec d'autres espèces}

Espèce souvent dominante par sa taille, elle n'est pas pour autant très abondante, quelques pour cent des communautés de batraciens. Les densités sont très variables de 8.8 à 45.8/ha d'étangs, de 6.6 à 119/km de canaux (BURY et WHELAN, 1984).

On considère qu'elle a réduit des populations de $R$. pipiens, $R$. blairi dans certaines zones du Colorado, éliminé $R$. pipiens fisheri dans le Nevada, déplacé et réduit $R$. aurora, $R$. boylii en Californie, qu'elle est en compétition avec $R$. pretiosa dans le Montana, etc. (BURY et WHELAN, 1984).

Le cas le plus étudié est probablement celui de $R$. aurora draytonii, dont la réduction en Californie serait due à l'introduction de $R$. catesbeiana (MOYLE, 1973). Mais le réexamen du problème par JENNINGS et HAYES (1985) montre que la prédation humaine, surtout sur les femelles, avait fait chuter la population à la fin du 19 ème siècle. C'est cette réduction qui avait poussé certains à l'introduction de $R$. catesbeiana au début des années 1900 , qui du reste n'est pas devenue très abondante par la suite.

En Italie, elle est accusée de faire disparaitre les espèces de grenouilles vertes autochtones du complexe esculenta dans certaines régions et pas dans d'autres (TOURATIER, 1992).

\section{Les risques potentiels en France}

Dans l'état actuel des connaissances, rien ne permet de dire que les grenouilles taureaux de la zone bordelaise aient un impact sur les écosystèmes, faute de mesures précises. Compte tenu de sa biologie, des données existantes concernant d'autres zones où elle a été introduite, on peut se poser les questions suivantes: 
- en tant que prédateur, a-t-elle une influence sur les populations de ses proies? En particulier au niveau des grenouilles locales, éventuellement des oiseaux (canetons).

- les têtards, dans les conditions naturelles, sont-ils des compétiteurs alimentaires d'autres groupes, comme les poissons ? Leur régime de détritus ne concerne en général guère les poissons, mais éventuellement leurs proies (invertébrés). En revanche, ces têtards peuvent représenter une source de nourriture pour de nombreux poissons.

- la compétition avec les autres batraciens existe-t-elle localement? Les données concernant ce problème doivent être étudiées avec soin, car n'oublions pas que la relation de cause à effet entre l'arrivée de $R$. catesbeiana et la réduction de certaines espèces a été parfois exagérée (ex. : $R$. aurora en Californie, $R$. esculenta en Italie). Actuellement, un suivi régulier de la densité des populations du complexe esculenta dans l'ouest de la France montre une réduction régulière depuis $10-15$ ans en l'absence de $R$. catesbeiana (données non publiées).

- cette espèce peut-elle présenter un risque pathologique pour l'homme et les animaux en sachant que la maladie des pattes rouges est déjà présente chez les batraciens autochtones?

\section{LE CAS DES TAXONS DU COMPLEXE ESCULENTA NON PRÉSENTS EN FRANCE}

Le probleme concernant les introductions de grenouilles vertes européennes est totalement différent, il s'agit de fuites issues de stockage des importations en vivant et/ou des travaux de recherche et d'enseignement. Ces animaux sont morphologiquement, biologiquement et écologiquement très proches de ceux présents en France.

\section{Rappel sur les grenouilles vertes d'Europe}

Les grenouilles vertes européennes présentent des caractéristiques génétiques particulières. Pour prendre l'exemple du complexe esculenta, le mieux connu, jusqu'aux années 60, trois morphotypes étaient connus : esculenta, lessonae, ridibunda. A la suite d'une série de travaux effectués en Pologne (biométrie, croisements dirigés, suivi des générations, etc.), l'équipe de BERGER (nombreuses publications depuis 1966) démontre que $R$. esculenta, la grenouille comestible de LINNE, est en fait un hybride naturel entre deux entités spécifiques bien définies : $R$. lessonae et $R$. ridibunda. Les résultats seront par la suite confirmés par des approches enzymatiques. $R$. esculenta présente du reste toutes les caractéristiques d'un hybride entre les deux espèces, tant au niveau morphologique qu'écologique. Ainsi, en général, l'autocroisement ne donne pas de descendance, les mortalités se produisent soit dès l'embryon, soit au niveau des têtards, quelquefois au niveau des grenouillettes. Mais cet hybride est un peu particulier, car il peut se maintenir par rétrocroisement avec une des deux espèces parentales. La descendance est alors essentiellement constituée d'hybrides grâce à un processus d'hybridogenèse qui élimine un génome parental au cours de la méiose. Des études récentes montrent cependant quelques exceptions, avec le développement d'une autre stratégie de l'hybride pour acquérir de la fertilité à partir de mâles triploïdes et de femelles diploïdes (REGNIER et NEVEU, 1986 ; GRAF et POLLS PELAZ, 1989 ; GÜNTHER et PLÖTNER, 1990).

Au fil des années, grâce au progrès des moyens d'analyses, des grenouilles classées sous le vocable ridibunda se sont révélées être des taxons différents (sur le plan biochimique, biométrique, écologique) suivant les régions.

Au niveau de leur écologie, toutes ces grenouilles sont aquatiques, avec des têtards détritiphages et des vitesses de croissance comparables, les tailles finales pouvant être légèrement différentes. Ainsi, les ridibunda sont plus grosses que les lessonae à l'état adulte. Des différences écologiques existent aussi : les ridibunda préfèrent les plans d'eau importants, les lessonae les marais et étangs riches en végétation. Les hybrides sont éclectiques, ainsi les esculenta se trouvent partout mais en abondance plus forte en étang (tendance ridibunda) qu'en marais (tendance lessonae). Enfin, certains taxons se répartissent dans des zones géographiques bien délimitées ( $R$. shqiperica, $R$. epeirotica). 
Au niveau de la France, on trouve deux grands complexes : au nord d'une ligne approximative La Rochelle-Nice se trouve avant tout le complexe esculenta, au sud le complexe perezi. Le premier est structuré principalement autour de l'association esculenta-lessonae dont l'abondance réciproque change avec les habitats (REGNIER et NEVEU, 1986). Mais à l'est se trouvent quelques associations esculenta-ridibunda sans parler des peuplements purs de ridibunda. Le deuxième complexe associe $R$. perezi et son hybride $R$. grafi (nomenclature : CROCHET et al., 1995).

\section{Le problème de l'importation des grenouilles vertes}

Les importations de grenouilles pour la consommation humaine se sont fortement développées dans les années 70-80. Elles concernent deux types de marchés : d'une part celui des surgelés avec des cuisses importées surtout d'Extrême-Orient (3000-4000 t/an) vendues en grandes surfaces pour l'essentiel, d'autre part celui des cuisses fraîches qui sont préparées à partir d'animaux importés vivants issus de captures dans différents pays de l'Europe du sud (800-900 t/an) destinés aux marchés traditionnels et à la restauration (NEVEU et REGNIER, 1985). Dans cette dernière filière, les grenouilles circulent en camion réfrigéré et sont stockées chez des grossistes, en attendant leur abattage. Les risques de fuites augmentent au cours des différentes manipulations et des îlots de colonisation peuvent se trouver autour de ces entreprises. Mais une autre source de fuites concerne l'enseignement où les séances de travaux pratiques peuvent donner lieu à des lâchers volontaires ou non. La colonisation du Campus de Rennes par des ridibunda est un exemple, il reste à faire une enquête auprès des autres universités... Enfin, il y a des lâchers volontaires par des personnes voulant repeupler ou faire des essais d'élevage.

Ces importations en vivant portent avant tout sur le type « ridibunda "lato sensu, compte tenu des pays d'origine. Il est ainsi probable que les importations de Yougoslavie (importantes de 1960 à 1980) aient introduit des R. shqiperica, celles d'Albanie (depuis 1980) et de Grèce des $R$. epeirotica. L'augmentation depuis les années 80 des importations de Turquie (200-300 t/an) et d'Egypte ne fait que rendre plus probable des implantations de diverses ridibunda et de $R$. bedriagae.

Quelques analyses récentes montrent une probable présence de géniteurs égyptiens dans la région lyonnaise (PAGANO, 1995) et de souches turques dans l'île de Ré (données non publiées). La présence de ridibunda à Roscoff, à Rennes au sein d'une vaste région à lessonaeesculenta, laisse penser à des introductions allochtones.

\section{Les risques potentiels en France}

Il est difficile de parler de " risques " dans la mesure où il faudrait déjà avoir des moyens de détection plus efficaces de ces souches étrangères. Les moyens actuels (biométrie, enzymes, $A D N$ ) restent à améliorer.

On peut certes envisager une sorte de " pollution génique " qui pourrait perturber, a priori, les populations locales. Comme beaucoup d'importations portent sur des animaux de climats plus cléments, il faudrait déjà s'assurer qu'ils supportent le climat français. L'introduction de certains gènes pourrait alors réduire la résistance des espèces locales par exemple à l'hiver. Mais le premier problème à résoudre est de savoir si les hybridations sont possibles et fertiles.

Ce qui semble le plus probable dans l'état actuel des connaissances, c'est l'installation directe d'une souche allochtone au niveau local, comme par exemple ridibunda sur le Campus de Rennes. Mais différentes investigations autour de la ville, au cours de la décennie, $n$ 'ont pas permis de retrouver ces animaux au sein des populations locales de lessonae-esculenta. II est cependant possible d'envisager dans ce cas d'école qu'une souche étrangère s'installe, entre en compétition avec les autochtones et les élimine. Actuellement, les connaissances écologiques sont trop succinctes pour conclure.

Finalement, le risque principal est de perturber l'ordonnancement des taxons au niveau géographique et d'augmenter les difficultés pour les spécialistes de l'analyse des structures génétiques des populations autochtones. 


\section{CONCLUSIONS GÉNÉRALES}

Dans le cadre des connaissances actuelles, le problème des introductions des autres taxons européens de grenouilles vertes semble présenter moins de danger que l'introduction de la grenouille taureau. II faut cependant veiller à éviter des repeuplements allochtones pour maintenir les structures géniques en place et mieux comprendre les causes réelles de la régression des stocks.

Pour la grenouille taureau, son aire actuelle en France semble très limitée mais demande cependant à être précisée. Ainsi, une action de réduction serait encore possible (destruction des oeufs). La présence de cette espèce peut en effet être considérée, a priori, comme néfaste, surtout du fait de la taille de l'animal et de son rôle de prédateur. II reste à mesurer son impact éventuel, dans la mesure où historiquement il a été souvent surestimé. Comme dans bien des cas lorsqu'il y a problème, on regarde rapidement l'allochtone comme cause principale de la perturbation. De même, il ne faut pas confondre "présence " avec "pullulation" et "extension géographique " avec "invasion". Enfin, la tendance générale est toujours de voir l'introduction d'une espèce uniquement sous ses aspects négatifs, et il y a effectivement de nombreux exemples dans ce sens, mais peut-être faut-il voir aussi quelques aspects positifs. Au niveau de la grenouille taureau par exemple, ses cuisses sont très appréciées et cette espèce pourrait être considérée comme une nouvelle ressource halieutique, pour peu que des études approfondies aient démontré son innocuité sur les écosystèmes en place.

Dans le cas contraire, il serait temps d'agir tant que son aire de répartition est limitée, par exemple par une destruction des pontes.

\section{BIBLIOGRAPHIE}

ALBERTINI G., LANZA B., 1987. Rana catesbeiana Shaw, 1802 in Italy. Alytes, 6, 117-129.

BURY R., WHELAN J.A., 1984. Ecology and management of the bullfrog. U.S. Dept. Int., Fish and Wildife Service, Res. Publ. 155, 1-23.

CARR A.H., AMBORSKI R.L., CULLEY D.D., AMBORSKI G.F., 1976. Aerobic bacteria in the intestinal tracts of bullfrogs (Rana catesbeiana) maintained at low temperatures. Herpetologica, 32, 239-244.

CROCHET P.A., OHLER A., TUNNER H., 1995. Rana (Pelophyllax) ridibunda Pallas, 1771, Rana (pelophyllax) perezi Seoane, 1885 and their associated klepton (Amphibia, Anura) : morphological diagnoses and description of a new taxon. Bul. Mus. nat. Hist. nat., Paris, $17,11-30$.

EMLEN S.T., 1968. Territoriality in the bullfrog Rana catesbeiana. Copeia, 240-243.

GRAF J.D., POLLS PELAZ M., 1989. Evolutionary genetics of the Rana esculenta complex. In DAWLEY R.M. et BOGART J.P. Eds, Evolution and Ecology of unisexual vertebrates. New York State Mus. Bull., 466, 289-302.

GÜNTHER R., PLÖTNER J., 1990. Making pattern in pure hybrid populations of water frogs, Rana kl. esculenta (Anura, Ranidae). Alytes, 8, 90-98.

HOWARD R.D., 1978. The influence of male defended oviposition sites on early embryo mortality in bullfrogs. Ecology, 59, 789-798.

JENNINGS M.R., HAYES M.P., 1985. Pre-1900 overharvest of California red-legged frogs (Rana aurora draytonii) : the inducement for bullfrog (Rana catesbeiana) introduction. Herpetologica, 41, 94-103.

MOYLE P.B., 1973. Effects of introduced bullfrogs Rana catesbeiana on the native frogs of the San Joaquin Valley, California. Copeia, 18-22. 
NEVEU A., REGNIER V., 1985. Une ressource halieutique mal connue : les grenouilles. Les problèmes liés à une véritable gestion des stocks. Bull. Fr. Pêche Piscic., 297, 35-47.

PAGANO A., 1995. Les complexes hybridogénétiques de grenouilles vertes de la plaine alluviale médio-Rhodanienne. Rapt. DEA, Univ. Lyon, $31 \mathrm{p}$.

REGNIER V., NEVEU A., 1986. Structure spécifique des peuplements en grenouilles du complexe Rana esculenta de différents milieux de l'ouest de la France. Acta Oecologica, Oecol. Applic., 7, 3-26.

STUMPEL A.H.P., 1992. Successful reproduction of introduced bullfrogs Rana catesbeiana in northwestern Europe : a potential threat to indigenous amphibians. Biol. Cons., 60, 61-62.

TOURATIER L., 1992. Première apparition en France (Région aquitaine) d'une grenouille géante américaine : Rana catesbeiana, en voie d'acclimatement. Intérêt zoologique et impact éventuel sur l'environnement. Bull. Soc. Vet. Prat. de France, 76, 219-228. 